\title{
Lattice bosons in a quasi-disordered environment: The effects of a superlattice potential on single particle and many particle properties
}

\author{
R. Ramakumar ${ }^{1}$, A. N. Das ${ }^{2}$, and S. $\mathrm{Sil}^{3}$ \\ ${ }^{1}$ Department of Physics and Astrophysics, \\ University of Delhi,Delhi-110007, India \\ ${ }^{2}$ Saha Institute of Nuclear Physics, \\ 1/AF Bidhannagar, Kolkata-700064, India and \\ ${ }^{3}$ Department of Physics, Visva Bharati, Santiniketan-731235, India
}

(Dated: 15 April 2015)

\begin{abstract}
In this paper we present a theoretical investigation of the effect of a superlattice potential on some properties of non-interacting bosons in one dimensional lattices with Aubry-Andŕe disorder potential. In the first part, we investigate the single particle localization properties. We find a re-entrant localization-delocalization transition and the development of multiple mobility edges for a range of superlattice potential strengths. In the second part, we study the Bose-Einstein condensation with an additional harmonic trapping potential. We find that an increase in the superlattice potential leads to an increase in the depletion of the condensate in the low temperature limit.
\end{abstract}

PACS numbers: PACS numbers: 03.75.Hh, 03.75.Lm, 37.10.Jk, 67.85.Hj,72.15.Rn 


\section{INTRODUCTION}

Studies of localization of the de Broglie waves in disordered and quasi-disordered environments have received renewed attention in the recent years primarily due to the direct observation of Anderson localization[1] of these matter waves in cold atom experiments [2, 3, 4]. While random disorder localizes all states in one dimension [5], a deterministic disorder distribution like the one in the Aubry-Andre model(AA) 6] leaves all states extended if the disorder strength remains below a critical value. The AA model[[] (or the Harper model[7]) has already been experimentally realized and a detailed study of its localization properties conducted [8, 9]. In the AA model, since all the states become localized beyond a critical disorder strength, there is no mobility edge. Recent theoretical studies [10, 11, 12, 13, 14, 15] have discovered the development of mobility edges in several extended AA models. Continuing along this direction of research, in this paper we consider the effects of a superlattice potential on the single particle and collective properties of non-interacting bosons in a one dimensional AA model. Among other results, we find a re-entrant single particle localizationdelocalization transition with increasing superlattice potential strength.

The rest of this paper is organized as follows. The studies of the single particle localization properties are presented in Sec. II. The Sec. III deals with the effects of the superlattice potential on the the Bose-Einstein condensation. The conclusions are given in Sec. IV.

\section{THE CHANGES IN THE LOCALIZATION PROPERTIES DUE TO THE SU- PERLATTICE POTENTIAL}

In this section, we consider the effect of a superlattice potential on the single particle localization properties. Consider a lattice boson moving in a one-dimensional lattice with AA disorder and an additional superlattice potential. For this system, the Hamiltonian is:

$$
H=-t \sum_{<i j>}\left(c_{i}^{\dagger} c_{j}+c_{j}^{\dagger} c_{i}\right)+\sum_{i}\left[\lambda \cos (2 \pi q i)+(-1)^{i} V\right] c_{i}^{\dagger} c_{i},
$$

where $t(>0)$ is the the energy gain when a boson hops from site $i$ to its nearest neighbor

$(\mathrm{NN})$ site $j, c_{i}^{\dagger}$ is a creation operator of a boson at site $i, \lambda$ the strength of the AA potential, $q$ $=(\sqrt{5}+1) / 2$ is the incommensurability parameter, and $V$ is the strength of the superlattice potential. Here $t, \lambda$, and $V$ have energy units. All the energies in this paper are measured 
in units of $t$ and all the lengths are measured in units of the lattice parameter. When $V$ $=0$, one obtains the AA model and energy independent localization of all the states for $\lambda>2$, and thus there is no mobility edge. To study the effects of finite $V$ on the localization properties, we first write $H$ in the single particle site basis and then numerically diagonalize it to obtain its eigen-energies and eigen-functions. All our results presented in this paper are for a lattice of 610 sites. We monitor the localization of a given eigen-state with amplitude $a_{i}$ at site $i$

$$
|\psi\rangle=\sum_{i} a_{i}|i\rangle
$$

by calculating its Inverse Participation Ratio (IPR) defined by

$$
I P R=\frac{\sum_{i} p_{i}^{2}}{\left(\sum_{i} p_{i}\right)^{2}},
$$

where,

$$
p_{i}=|<i| \psi>\left.\right|^{2}=\left|a_{i}\right|^{2}
$$

In Fig. 1 we have displayed the IPR values of all the 610 states for various values (< 2 ) of $\lambda$. For these values of $\lambda$, all the states are extended in the absence of $V$. We find that increasing $V$ drives most states to localized. We also notice that the band edge states are more susceptible to localization and become localized for smaller values of $V$. When we increase $\lambda$ another qualitatively different behavior is found. In the results shown for $\lambda$ $=1.3$ and 1.5, we notice that some states exhibit a re-entrant localization-delocalization transition. We do not find any re-entrant behavior with increasing $\lambda$ for a fixed $V$ as shown in Fig. 2. A consequence of the localization-delocalization transition is the enhancement of the entanglement with increasing $V$ for the concerned states in relevant regions, as shown in Fig. 3. The entanglement is measured by the Shannon entropy [16] defined by

$$
S=-\sum_{i} p_{i} \log _{2} p_{i}
$$

The variation of the single particle spectrum with $V$ for $\lambda=1.5$ is shown in Fig. 4 . It is clear that for several values of $V$ the system develops multiple mobility edges. This may be contrasted with the development of a single mobility edge due next nearest neighbor (NNN) hopping in the absence of $V[10,11,12,13]$. The development of mobility edges due to $V$ can also be seen in Fig. 5, where we have plotted the single particle spectrum and the corresponding IPR values as a function of $\lambda$ for $V=0.5$. One also notices that beyond 


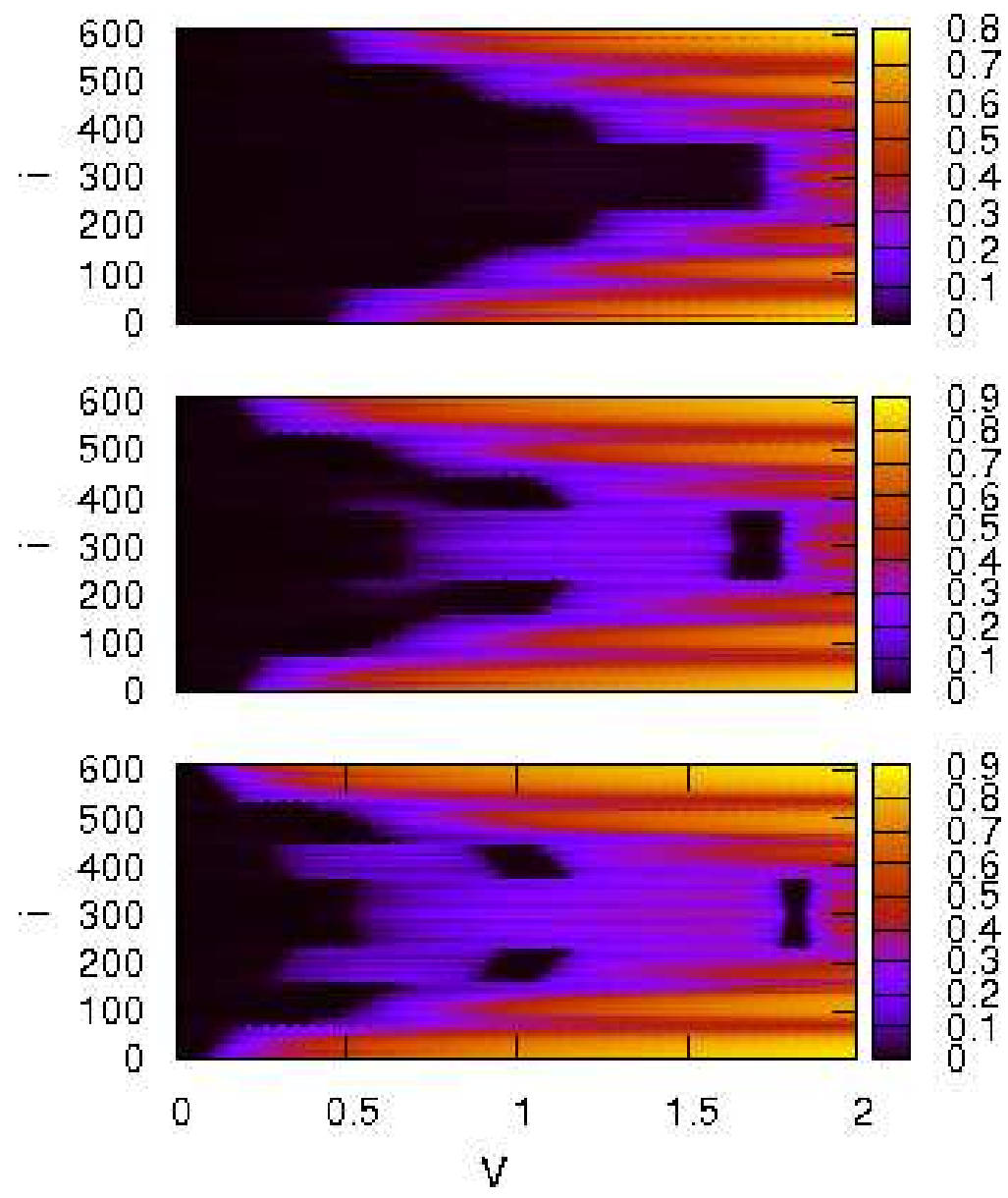

FIG. 1: The IPR values (in color) for different energy eigen-states as a function of $\mathrm{V}$ for $\lambda=1.0$ (top panel), 1.3 (middle), and 1.5 (bottom) for a closed chain of 610 sites.

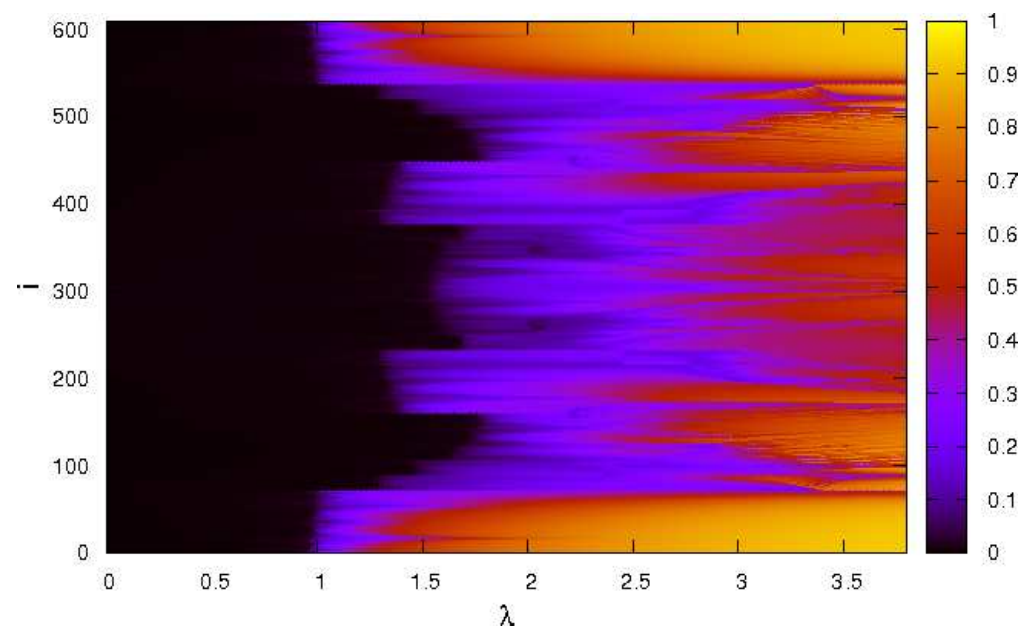

FIG. 2: The IPR values (in color) for different energy eigen-states as a function of $\lambda$ for $\mathrm{V}=0.5$ and for a closed chain of 610 sites. 


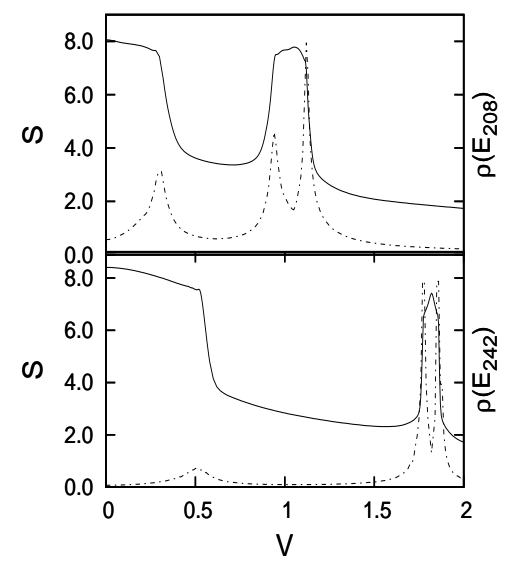

FIG. 3: The variation of the entanglement $S$ (solid line) with V of the eigen-energy levels 208 (top panel) and 242 (bottom panel) for $\lambda=1.5$. The variations of the density of states (DOS) $\left(\rho\left(E_{208}\right)\right.$ and $\left.\rho\left(E_{242}\right)\right)$ for these eigen-energy levels are also shown (dash-dot).

$\lambda>2$, all the states are localized. Further, while $\lambda$ shifts more levels in to the central gap region, the $V$ has the opposite effect. Note also the development of multiple mobility edges in some range of values of $\lambda$. We also find that the delocalization to localization transition or vice versa for any energy eigen-state is associated with a rise in the single particle density of states (DOS). The variations of the DOS with $V$ for two eigen-energies are also shown in Fig. 3. It is seen that whenever $S$ drops or rises rapidly signaling a delocalizationlocalization transition or vice versa the DOS shows a peak. At this juncture we are unable to provide an analytical explanation of these results. Clearly, the superlattice potential destroys the self-duality of the AA model, and a complex interplay of the AA quasi-disorder and the superlattice potential lead to new qualitative features like the re-entrant localizationdelocalization transition and the development of multiple mobility edges. Considering cold atom experiments in mind, we have checked the effect of an overall harmonic confining 


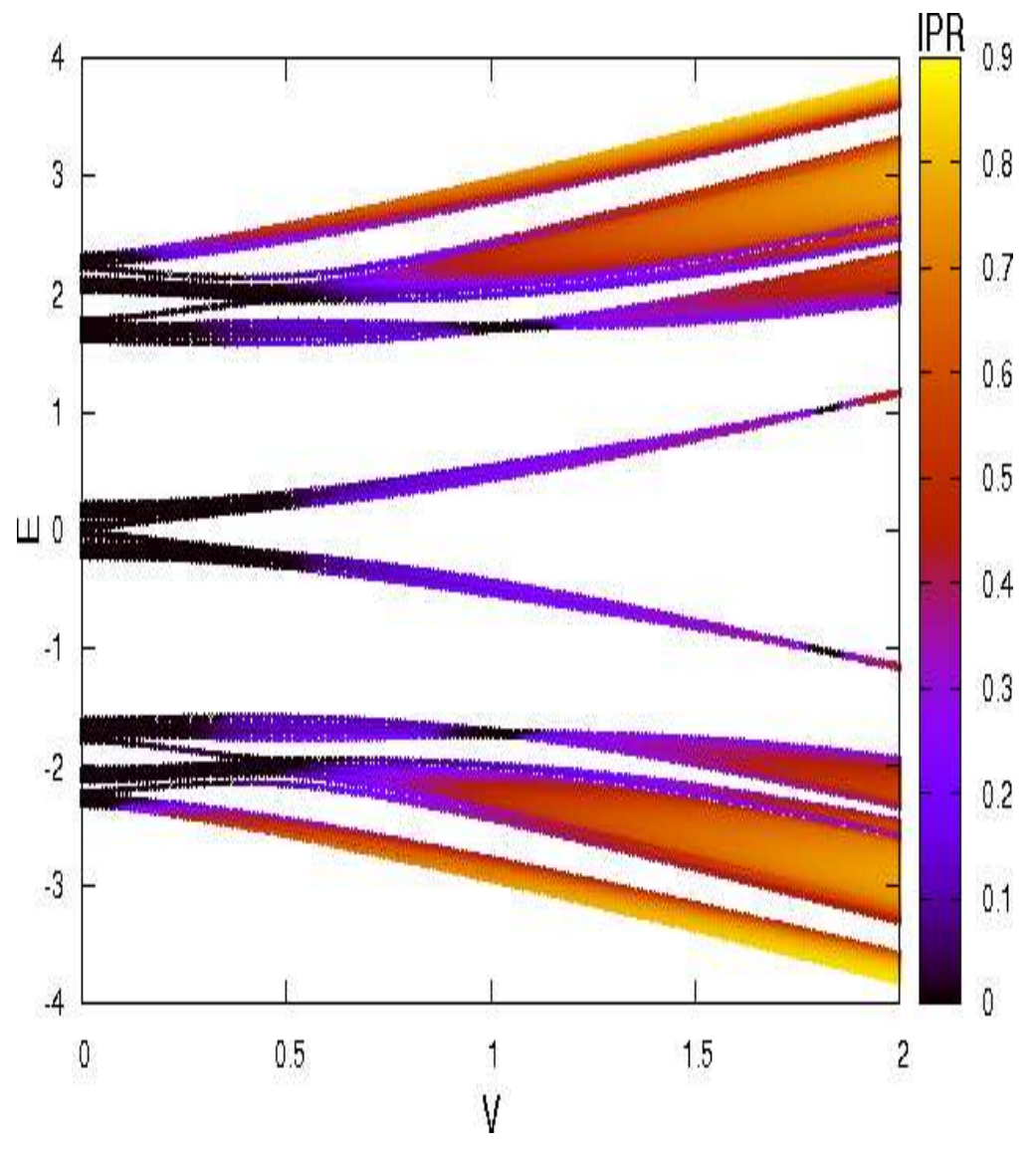

FIG. 4: The single particle spectrum as a function of $\mathrm{V}$ for $\lambda=1.5$ for a closed chain of 610 sites. The IPR values of the corresponding states are shown in color.

potential. In this case the system Hamiltonian becomes

$$
\tilde{H}=H+\sum_{i} k i^{2} c_{i}^{\dagger} c_{i}
$$

where $k$, which has an energy unit, is the harmonic confining potential strength. For the harmonic trap, we use open boundary conditions which is more appropriate. The results of these calculations are shown in Fig. 6. The confining potential clearly drives more states localized. This behavior is qualitatively expected as a confining potential generally favors localization. 


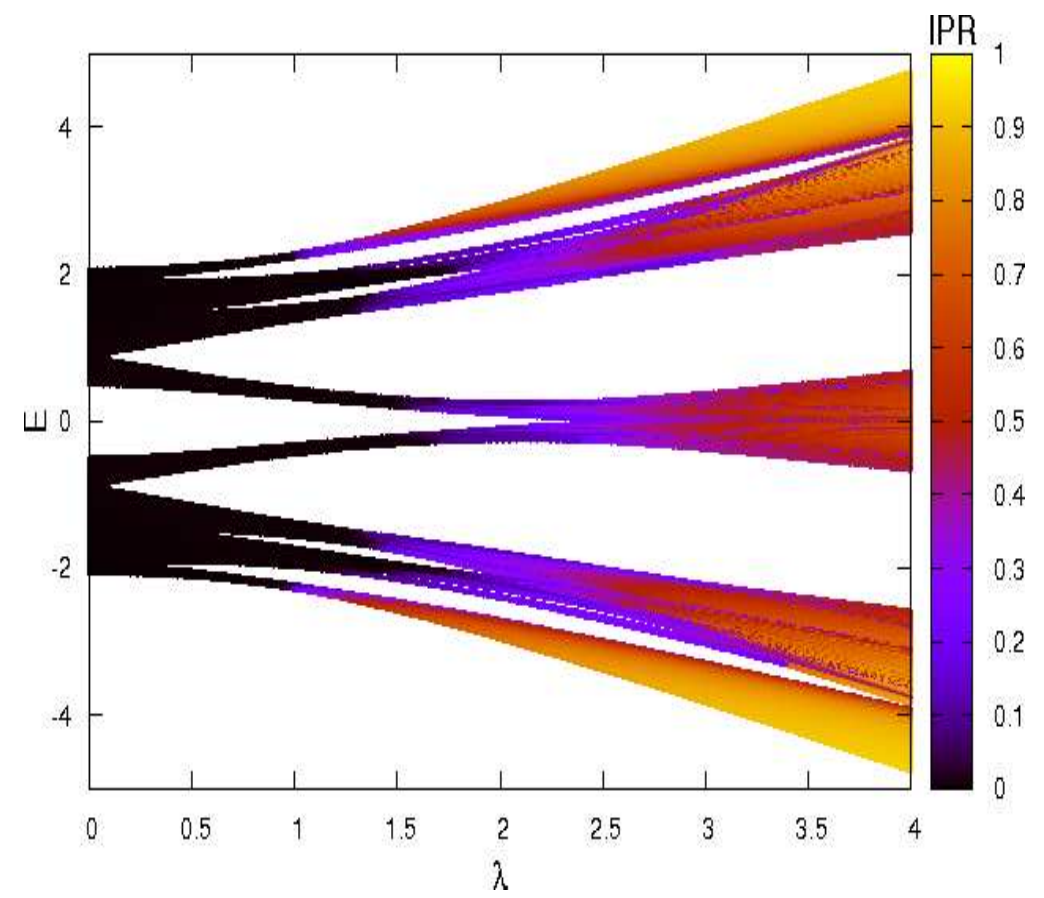

FIG. 5: The single particle spectrum as a function of $\lambda$ for $\mathrm{V}=0.5$ for a closed chain of 610 sites. The IPR values are shown in color.

\section{THE INFLUENCE OF THE SUPERLATTICE POTENTIAL ON THE BOSE- EINSTEIN CONDENSATION}

In the previous section we have shown the superlattice potential leads to significant changes in the nature of the eigen-states of the AA model. These changes may influence the collective properties like Bose-Einstein condensation of a many-boson system (see also the note in Ref. 17). In this section, we study the effect of $V$ on the ground state occupancy of a many-boson system in AA potential with an overall harmonic confining potential. The Hamiltonian of this system of lattice bosons is then:

$$
\tilde{\tilde{H}}=\tilde{H}-\mu \sum_{i} c_{i}^{\dagger} c_{i}
$$

where $\mu$ is the chemical potential. The boson number equation is

$$
N=\sum_{i=1}^{m} N\left(E_{i}\right),
$$

where $E_{i}$ 's are the energy levels obtained by diagonalizing $\tilde{H}$ and

$$
N\left(E_{i}\right)=\frac{1}{e^{\beta\left(E_{i}-\mu\right)}-1},
$$




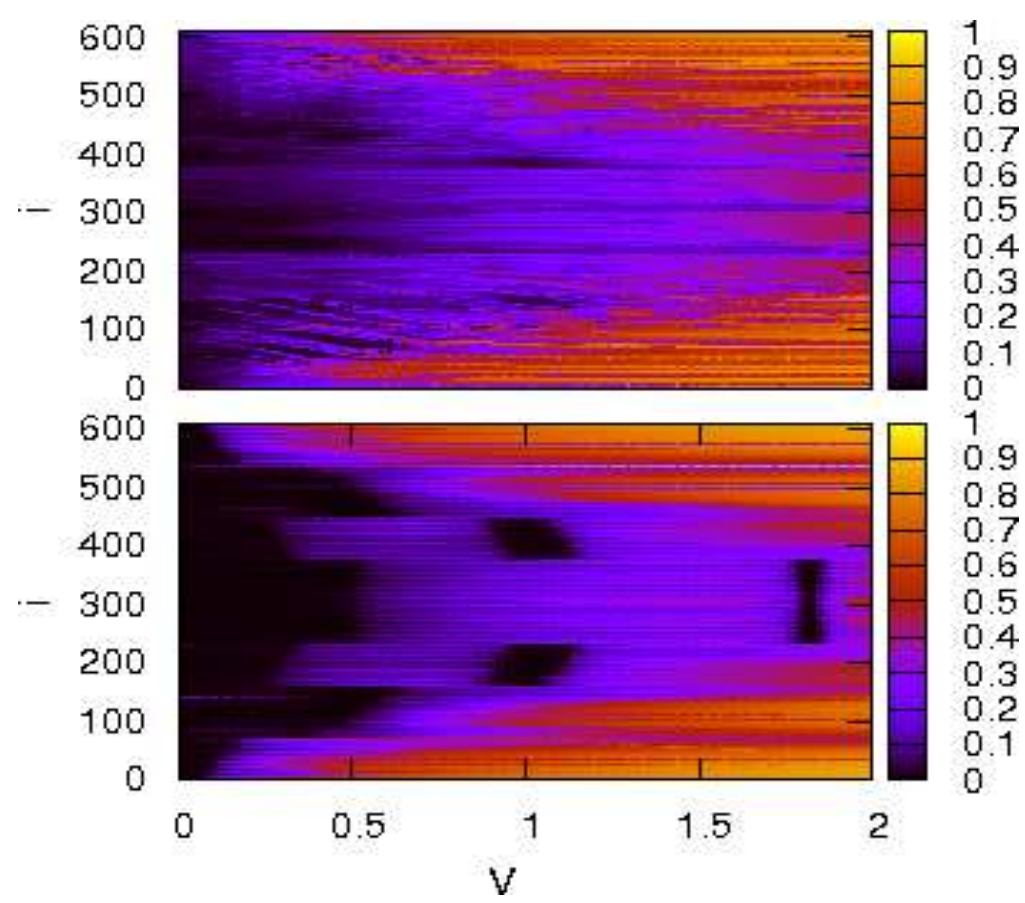

FIG. 6: The effect of a harmonic potential on the IPR values (in color) for different energy eigenstates as a function of $\mathrm{V}$ for $\lambda=1.5$ for an open chain of 610 sites. The results are shown for $\mathrm{k}=$ 0.00001 (top panel) and for $\mathrm{k}=0$ (bottom panel). The harmonic potential is placed symmetrically about the center of the lattice.

where $\beta=1 / k_{B} T$ in which $k_{B}$ is the Boltzmann constant and $T$ the temperature. For a fixed boson number and temperature, the chemical potential and then the boson populations in various energy levels are calculated using the boson number equation. The temperature variation of the fractional ground state population $\left(N_{0} / N\right)$, where $N_{0}=N\left(E_{1}\right)$ is the ground state population and $N$ the total number of bosons, for various values of $V$ and $\lambda=1.5$ is shown in Fig. 7. While in the low temperature range $\left(k_{B} T<0.1\right)$, the $N_{0} / N$ decreases with $V$, it increases with $V$ in the higher temperature range. The behavior of $N_{0} / N$ is determined by the low energy levels. We examined the $V$ dependence of the energies of the lowest few excited states. The results for $\lambda=1.5$ are shown in Fig. 8. The first excited state move close to the ground state with increasing $V$ leading to a rapid thermal depletion of the ground state. Beyond $V>0.2$, the ground state and the first excited state become almost degenerate and the bosons are distributed equally in these levels leading to a drop of $N_{0} / N$ from 1 to about 0.5 as seen in Fig. 7. We also see that the second excited state onwards, the excited state energies move away from the ground state with increasing $V$. 


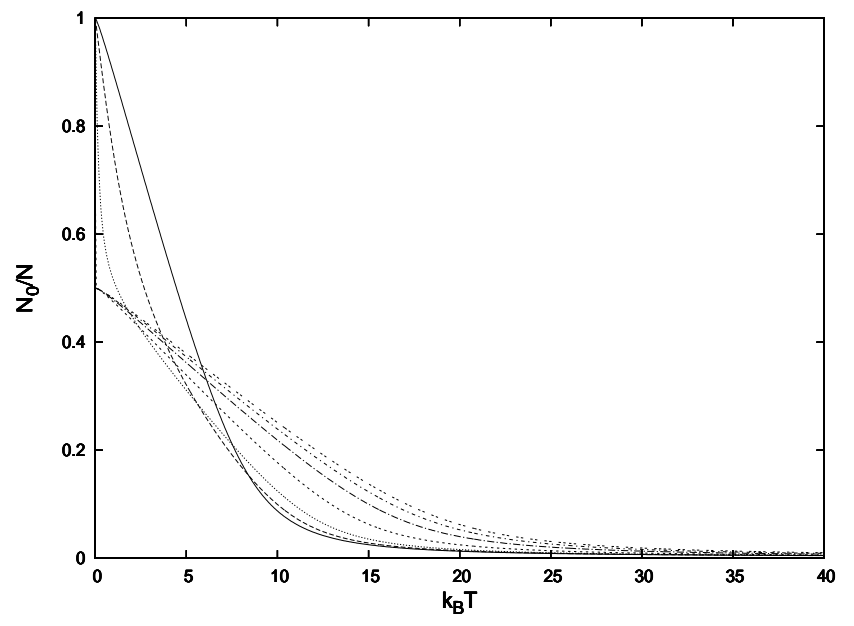

FIG. 7: The variation of the condensate fraction with temperature for 10000 bosons in an open chain with 610 sites in a harmonic trap with $k=0.00001$ and $\lambda=1.5$. The results are for: $\mathrm{V}=0$ (solid line), 0.1 (long dash), 0.2 (dot), 0.5 (short dash), 1.0 (dash-dot), 1.5 (short dash-dot), and 2.0 (double dashes).

So, in the higher temperature range, the population of these excited states decrease with increasing $V$ leading to an increase in $N_{0} / N$.

\section{CONCLUSIONS}

In this paper, we first investigated the effect of a superlattice potential on the single particle localization properties of a lattice boson in the presence of AA disorder. We find a reentrant localization-delocalization transition and the development of multiple mobility edges for range of superlattice potential strengths. We also studied the Bose-Einstein condensation with an additional harmonic trapping potential. It is found that while an increase in the superlattice potential leads to an increase in the depletion of the condensate in the low temperature limit, it has the opposite effect beyond this temperature regime. These trends

of the condensate fraction was argued to be resulting from the changes in the low energy single particle spectrum produced by the superlattice and the quasi-disorder potentials. 


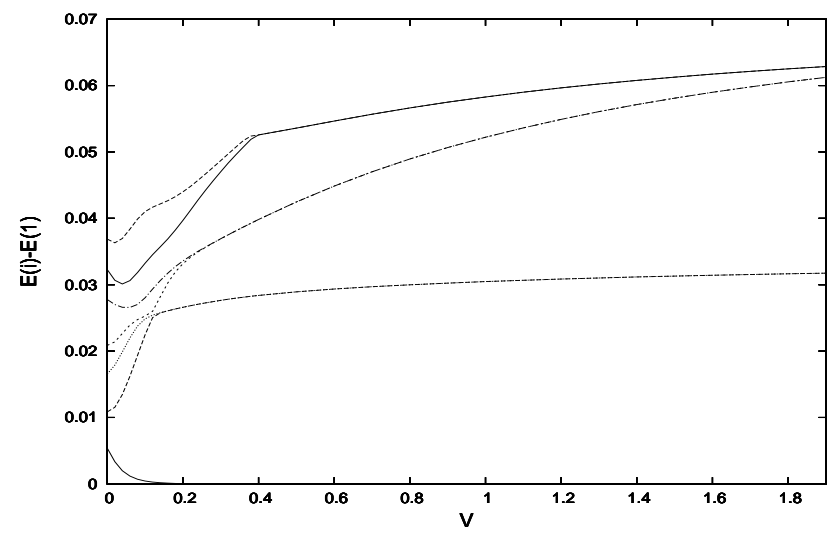

FIG. 8: The variation of the difference between energies of the ground state and first few excited states $E(i)-E(1)$ for (from bottom to top) $\mathrm{i}=2,3,4,5,6,7$, and 8 , for $\lambda=1.5$.

\section{Acknowledgments}

RRK thanks Professor B. K. Chakrabarti, Director, SINP and Professor R. Ranganathan, Head, CMP Division, SINP for hospitality at SINP.

[1] P. W. Anderson, Phys. Rev. 109, 1492 (1958).

[2] J. Billy, V. Josse, Z. Zuo, A. Bernard, B. Hambrecht, P. Lugan, D. Clément, L. SanchezPalencia, P. Bouyer, and A. Aspect, Nature 453, 891(2008).

[3] S. S. Kondov, W. R. McGehee, J. J. Zirbel, and B. DeMarco, Science 334, 66 (2011).

[4] F. Jendrzejewski, A. Bernard, K. Müller, P. Cheinet, V. Josse, M. Piraud, L. Pezzé, L. SanchezPalencia, A. Aspect, and P. Bouyer, Nature Phys. 8, 398 (2012).

[5] N. F. Mott and W. D. Twose, Adv. Phys. 10, 107 (1961).

[6] S. Aubry and G. Andŕe, Ann. Isr. Phys. Soc. 3, 133 (1980).

[7] P. G. Harper, Proc. Phys. Soc. London Sect. A 68, 874 (1955). See also M.Ya. Azbel, Sov. Phys. JETP 19, 634 (1964) and Phys. Rev. Lett. 43, 1954 (1979).

[8] G. Roati, C. D'Errico, L. Fallani, M. Fattori, C. Fort, M. Zaccanti, G. Modugno, M. Modugno, and M. Inguscio, Nature 453, 895 (2008). 
[9] Y. Lahini, R. Pugatch, F. Pozzi, M. Sorel, R. Morandotti, N. Davidson, and Y. Silberberg, Phys. Rev. Lett. 103, 013901 (2009).

[10] D. J. Boers, B. Goedeke, D. Hinrichs, and M. Holthaus, Phys. Rev. A 75, 063404 (2007).

[11] J. Biddle, B. Wang, D. J. Priour, Jr., and S. Das Sarma, Phys. Rev. A 80, 021603(R) (2009).

[12] J. Biddle and S. Das Sarma, Phys. Rev. Lett. 104, 070601 (2010).

[13] J. Biddle, D. J. Priour, Jr., B. Wang, and S. Das Sarma, Phys. Rev. B 83, 075105 (2011).

[14] P. Rebeiro, M. Haque, and A. Lazarides, Phys. Rev. A 87, 043635 (2013).

[15] R. Ramakumar, A. N. Das, and S. Sil, Physica A 401, 214 (2014).

[16] J. -M. Stéphan, S. Furukawa, G. Misguich, and V. Pasquier, Phys. Rev. B 80, 184421 (2009).

[17] While a uniform bose gas cannot undergo Bose condensation in one dimension (1d), it is possible in the inhomogeneous case (for bosons in harmonic potentials see: W. Ketterle, N. J. van Druten, Phys. Rev. A 54, 656 (1996) for the continuum case and R. Ramakumar, A. N. Das, and S. Sil, Eur. Phys. J. D 42, 309 (2007) for the lattice case). In this paper the origin of inhomogeneities are the AA and the confining potentials. Though we do not consider random disorder, it is appropriate for us to mention some studies of Bose condensates in random disorder in 1d. These are: J. M. Luttinger and H. K. Sy, Phys. Rev. A 7, 712 (1973); P. Lugan, D. Clément, P. Bouyer, A. Aspect, M. Lewenstein, and L. Sanchez-Palencia, Phys. Rev. Lett. 98, 170403 (2007); L. Sanchez-Palencia, D. Clément, P. Lugan, P. Bouyer, G. V. Shlyapnikov, and A. Aspect, Phys. Rev. Lett. 98, 210401 (2007); I. N. de Oliveira, F. A. B. F. de Moura, R. A. Caetano, and M. L. Lyra, Phys. Rev. B 82, 172201 (2010). 